\title{
Obesity, colorectal cancer and MACC1 expression: A possible novel molecular association
}

\author{
INA BÄHR ${ }^{1}$, LINA JAESCHKE ${ }^{2}$, KATHARINA NIMPTSCH $^{2}$, JÜRGEN JANKE ${ }^{2-4}$, PIA HERRMANN $^{5}$, \\ DENNIS KOBELT ${ }^{5}$, HEIKE KIELSTEIN ${ }^{1}$, TOBIAS PISCHON ${ }^{2,4-6^{*}}$ and ULRIKE STEIN ${ }^{5,7 *}$ \\ ${ }^{1}$ Institute of Anatomy and Cell Biology, Medical Faculty of Martin Luther University Halle-Wittenberg, \\ D-06108 Halle (Saale); ${ }^{2}$ Max-Delbrück-Center for Molecular Medicine in The Helmholtz Association,
}

Molecular Epidemiology Research Group; ${ }^{3}$ Max-Delbrück-Center for Molecular Medicine in The Helmholtz Association,

Biobank Technology Platform, D-13125 Berlin; ${ }^{4}$ Berlin Institute of Health at Charité-Universitätsmedizin Berlin,

Biobank Core Facility, D-10117 Berlin; ${ }^{5}$ Translational Oncology of Solid Tumors, Experimental and Clinical Research Center,

Charité-Universitätsmedizin Berlin and Max-Delbrück-Center for Molecular Medicine in The Helmholtz Association,

D-13125 Berlin; ${ }^{6}$ Charité-Universitätsmedizin Berlin, Corporate Member of Freie Universität Berlin and

Humboldt-Universität zu Berlin, D-10117 Berlin; ${ }^{7}$ German Cancer Consortium (DKTK), D-69120 Heidelberg, Germany

Received October 21, 2021; Accepted December 6, 2021

DOI: 10.3892/ijo.2022.5307

\begin{abstract}
Obesity is a major and increasing public health concern, associated with an increased risk of and mortality from several types of cancer including colorectal cancer (CRC), being associated with cancer progression, metastasis and resistance to therapy. It was hypothesized that the expression of cancer/metastasis-inducing gene metastasis-associated in colon cancer 1 (MACC1) is increased in obesity, which may constitute a link to obesity-induced cancer. The present study thus analyzed circulating cell-free plasma MACC1 expression levels in human obese (vs. normal weight) adult individuals from independent studies, namely the Martin Luther University (MLU) study $(n=32)$ and the Metabolic syndrome study (MetScan, Berlin) $(n=191)$. Higher plasma MACC1 levels were found in obese individuals, increasing with a greater body fat mass and body mass index; these levels were predominantly observed in male and to a lesser extent in female individuals, although the results were not significant. A reduction in body fat mass following dietary intervention and physical exercise decreased the MACC1 expression levels in the MLU study. Furthermore, Wistar rats with diet-induced
\end{abstract}

Correspondence to: Professor Ulrike Stein, Translational Oncology of Solid Tumors, Experimental and Clinical Research Center, Charité-Universitätsmedizin Berlin and Max-Delbrück-Center for Molecular Medicine in The Helmholtz Association, 10 Robert-Rössle-Strasse, D-13125 Berlin, Germany E-mail: ustein@mdc-berlin.de

\section{*Contributed equally}

Key words: obesity, colorectal cancer, MACC1 expression, plasma, human subjects, animals obesity exhibited slightly increased plasma MACC1 levels compared with rats of normal weight. The obese Wistar rats exposed to azoxymethane to induce colon cancer exhibited a more severe colon tumor outcome, which was associated with significantly increased MACC1 levels compared with their non-obese littermates. On the whole, the findings of the present study suggest an association between MACC1 and obesity, as well as with obesity-induced CRC.

\section{Introduction}

Obesity is a major and continually increasing public health concern. Since 1975, the worldwide prevalence of obesity has rapidly increased almost 3 -fold (1). Obesity is a public health concern in developed and developing countries, in all ages and in all socioeconomic groups. The World Health Organization (WHO) published in 2016 that 1.9 billion adults (aged $\geq 18$ years; $39 \%$ ) were overweight [body mass index $\left.(\mathrm{BMI}) \geq 25.0 \mathrm{~kg} / \mathrm{m}^{2}\right]$ and 650 million $(13 \%)$ were obese (BMI $\geq 30 \mathrm{~kg} / \mathrm{m}^{2}$ ) (2). For Germany, $67 \%$ of adult men are overweight and $23 \%$ obese, and $53 \%$ of women are overweight, with $24 \%$ being obese (3).

Obesity is linked to a number of severe and chronic diseases, such as type 2 diabetes, coronary heart disease or stroke. Moreover, obesity is also associated with an increased risk of and mortality from several types of cancer, including colorectal cancer (CRC), and is associated with cancer progression, metastasis and resistance to therapy (4-6). In fact, it has been estimated that a substantial proportion (up to 20\%) of the total cancer cases may be attributed to being overweight (BMI $\geq 25 \mathrm{~kg} / \mathrm{m}^{2}$ ) (7). CRC is the second most common cause of cancer-related mortality in the United States (8). Human studies have demonstrated that a body weight increase of $5 \mathrm{~kg}$ elevates the incidence of colon cancer by $6 \%$ (9). Moreover, a high BMI in patients with colon cancer has also been linked to an increased mortality rate $(10,11)$. 
A variety of factors may contribute to obesity-induced cancer, such as metabolic factors, microbiota or chronic inflammation (12). However, the underlying molecular pathophysiological mechanisms and signaling pathways are not yet entirely understood. The present study focused on the cancer- and metastasis-inducing gene, metastasis-associated in colon cancer 1 (MACC1), in the context of obesity and obesity-induced cancer. The authors previously newly discovered MACC1 in the tissues of patients with colon cancer $(13,14)$. The authors have previously demonstrated its functions for inducing phenotypes, such as proliferation, migration, invasion, dissemination, etc. in cell culture, as well as carcinogenesis and metastases in several mouse models $(13,15,16)$. Since the discovery of MACC1, the authors of the present study, as well as several other research groups have demonstrated the clinical significance of MACC1 as a causal, prognostic and predictive biomarker for $>20$ solid cancer types (14), including meta-analyses for solid cancers, cancers of the digestive system, colorectal, hepatocellular, gastric, gynecological and breast cancer (17-22). The causal contribution of MACC1 for rewiring cell metabolism has been demonstrated by the authors of the present study, as well as others (23-25). A recent study reported MACC1-driven metabolic networks in $\mathrm{CRC}$, such as rewiring glucose and glutamine metabolism, an increase in glucose use by the enhanced surface of glucose transporter 1 (GLUT1) or an increase in glutamine and pyruvate use by enhanced uptake (25). It was thus concluded that MACC1 is an important regulator of cancer metabolism.

The present study aimed to determine whether the plasma MACC1 levels may be increased in obesity, which may be a link to obesity-associated CRC. The levels of circulating cell-free MACC1 transcripts were first investigated in the blood of obese subjects from two independent human studies of adult individuals: One study of subjects from the Martin Luther University (MLU study) (26) and a second study on individuals from the Metabolic Syndrome study (MetScan) (27), thereby evaluating a potential link of MACC1 expression and BMI or body fat mass. These analyses were complemented with plasma MACC1 expression data from the MLU intervention study of subjects analyzed following 3 months of a moderate dietary and exercise program. Plasma MACC1 levels in Wistar rats fed a normal-fat diet (NFD) or a high-fat diet (HFD) were further compared. Finally, the animals were exposed to azoxymethane (AOM) in order to test the hypothesis of the induction of CRC in the context of obesity and MACC1 expression (28).

\section{Materials and methods}

Animal experiments. Animal experiments were performed as previously described $(27,28)$. All research and animal care procedures were approved by the local Animal Care Committee (reference no. 42502-2-1200 MLU). The principles of laboratory animal care were followed according to the guidelines of the European (FELASA) and German Society of Laboratory Animal Sciences (GV-SOLAS). In brief, male Wistar rats ( 6 weeks old, $n=50$, Charles River $\mathrm{GmbH}$ ) were randomly divided according to body weight into two groups. One group $(n=25)$ received a NFD (control, 4\% fat, C1090-10, Altromin) and the other group $(n=25)$ received the corresponding HFD (34\% fat, C1090-60, Altromin) for 46 weeks.
At 8 weeks following the start of dietary intervention, 12 (NFD group) or 11 (HFD group) rats were injected subcutaneously with $15 \mathrm{mg} / \mathrm{kg}$ body weight of the carcinogen, AOM (Sigma-Aldrich; Merck KGaA), once a week for 2 weeks to induce colon cancer growth. The corresponding control groups received a subcutaneous injection of $0.9 \%$ sodium chloride ( $\mathrm{NaCl}$; NFD: $\mathrm{n}=13$, HFD: $\mathrm{n}=14$ ). At 37 weeks after the final $\mathrm{AOM}$ injection, the rats were sacrificed by isoflurane inhalation anesthesia (1.5-2\% v/v in oxygen) followed by cardiac puncture for blood collection (8-9 $\mathrm{ml}$ per rat). The final body weight was determined directly prior to cardiac puncture. Visceral fat mass was removed and determined by weighing the total intra- and retroperitoneal adipose tissues. The colon was examined for macroscopically visible tumors and tumor-bearing areas were excised, followed by tumor classification according to histologic grade by an expert pathologist.

\section{Human populations}

Description of the MLU study. The MLU study was performed as previously described (26). In brief, male and female participants were recruited. The inclusion criteria were as follows: $\mathrm{BMI} \geq 30 \mathrm{~kg} / \mathrm{m}^{2}$ and an age between 18 and 65 years. The exclusion criteria were pregnancy, breastfeeding, chronic infections, acute diseases, metabolic or endocrine diseases (e.g., type 1 diabetes, hypo- or hyperthyroidism), or immunosuppression (e.g., chemotherapy). The participants ( $n=32$; male, $\mathrm{n}=14$; female, $\mathrm{n}=18$ ) were divided into 2 groups as follows: One experimental group ( $\mathrm{n}=19 ; 8$ males and 11 females) and one control group ( $\mathrm{n}=13 ; 6$ males and 7 females). The participants of the experimental group performed a moderate exercise training program of three 1 -h exercise sessions per week: One unit endurance training and two units of a combined weight and endurance training. The training intensity was adapted to the individual performance level determined before the start of intervention. In addition, participants of the experimental group received dietary recommendations according to the guidelines of the German Society for Nutrition to reduce their body weight through information flyers as well as individual or group consultations. Participants of the control group were instructed not to change their lifestyle and to maintain their weight. They received neither dietary advices nor an exercise training program.

The following parameters were determined before and at 3 months after the start of the intervention as previously described: Body weight and height with the calculation of BMI, waist and hip circumferences, as well as body fat mass measured by bioelectrical impedance analysis (26). In addition, blood plasma of participants was obtained from blood samples after fasting before and at 3 months following the start of intervention. Each participant signed informed consent prior to participation.

Description of the MetScan study. MetScan is a cross-sectional study that aimed to investigate to what extent abdominal volume, automatically determined using a 3D body surface scanner algorithm, allows metabolic characterization $(27,30)$. Following a standardized recruitment protocol, a total of 516 participants, aged 18-79 years, were recruited. The inclusion criteria were German language skills and an ability to provide informed consent. The exclusion criteria were an inability to 
perform a body scan measurement and any condition distorting body shape (e.g., casts).

Sex, age, and a history of diabetes mellitus and dyslipidemia were assessed in computer-assisted interviews. Body height (cm, stadiometer SECA 285, Hamburg, Germany), body weight and fat mass ( $\mathrm{kg}$, bioelectrical impedance analysis device SECA mBCA 515, SECA), as well as both waist and hip circumference (cm, measuring tape SECA 201, SECA) were measured with an accuracy of one decimal place by trained personnel according to WHO guidelines (31). BMI was calculated as body weight divided by the square of body height $\left(\mathrm{kg} / \mathrm{m}^{2}\right)$; waist-to-hip ratio (WHR) was calculated as the waist circumference divided by hip circumference; the waist-to-height ratio (WHtR) was calculated as the waist circumference divided by body height.

Blood pressure was calculated as the mean of the second and third blood pressure measurement taken after an initial 5-min rest (OMRON Medizintechnik mbH) (32). Blood samples were collected in BD Vacutainer ${ }^{\circledR}$ (BD Becton-Dickinson) tubes following a standardized venipuncture protocol. EDTA tubes $(9 \mathrm{ml})$ were centrifuged at $2,000 \mathrm{x} \mathrm{g}$ for $15 \mathrm{~min}\left(15^{\circ} \mathrm{C}\right)$ and the plasma supernatant was aliquoted and immediately frozen on dry ice and stored at $-80^{\circ} \mathrm{C}$ until the measurement of MACC1 levels.

Concentrations of triglycerides (TG, mmol/l), high-density lipoprotein cholesterol (HDL-C, $\mathrm{mg} / \mathrm{dl}$ ) in serum, glucose $(\mathrm{mmol} / \mathrm{l})$ in citrate fluoride plasma and hemoglobin A1c (HbAlc; $\mathrm{mmol} / \mathrm{mol})$ in EDTA blood were determined from blood samples following fasting ( $>8 \mathrm{~h}$ ) (Hospital Laborverbund Brandenburg-Berlin $\mathrm{GmbH}$ ).

Parameters of the metabolic syndrome (MetS) were classified according to the Harmonized model as follows (33) elevated TG concentration ( $\geq 1.7 \mathrm{mmol} / \mathrm{l}$ ); decreased HDL-C concentration (men, $<1.0 \mathrm{mmol} / \mathrm{l}$; women, $<1.3 \mathrm{mmol} / \mathrm{l}$ ); elevated blood pressure (systolic blood pressure $\geq 130 \mathrm{mmHg}$ or diastolic blood pressure $\geq 85 \mathrm{~mm} \mathrm{Hg}$ or reported diagnosis of hypertension); elevated glucose concentration ( $\geq 5.6 \mathrm{mmol} / \mathrm{l}$ or reported diagnosis of diabetes). If participants met at least three of the aforementioned criteria or elevated waist circumference (WC; men, $\geq 94 \mathrm{~cm}$; women, $\geq 80 \mathrm{~cm}$ ) this was defined as having MetS (33).

Selection of participants from the MetScan study. In the preliminary analyses derived from the MLU study, correlation coefficients of Rho=0.2-0.5 were observed for the association of MACC1 with fat mass and body weight. Assuming similar effect sizes and with the adjustment for two covariates (age and sex), a study sample size of 195 participants was determined to be necessary to detect correlation coefficients of Rho $>0.2$. Following the exclusion of participants with missing covariates, 429 MetScan participants were eligible for inclusion in the present analyses. From these, selected 204 participants were randomly, stratified by BMI categories (normal weight, $<25$; pre-obesity, 25-29.9; obesity, $\geq 30 \mathrm{~kg} / \mathrm{m}^{2}$ ) and sex. This resulted in 68 participants per BMI category, with 34 males and 34 females in the low and middle BMI category. The highest BMI category included slightly more females than males (41 vs. 27) as the number of males eligible in this category was limited. For these 204 participants, a blood sample was transferred to the Translational Oncology of Solid Tumors Group, Max Delbrück Center for Molecular Medicine in the Helmholtz Association (MDC), to determine the level of circulating cell-free MACC1 transcripts.

Determination of circulating cell-free MACC1 transcripts using reverse transcription-quantitative PCR (RT-qPCR)

$M L U$. The entire procedure, as employed for the MLU study, has already been described for patients suffering from CRC, gastric and ovarian cancer, and glioblastomas (34-38). Herein, total RNA was isolated from the plasma samples by employing the high pure viral RNA kit (Roche Diagnostics) according to the manufacturer's instructions. The analysis of circulating cell-free MACC1 transcripts was carried out with the hybridization probe detection format using amplicon-specific hybridization probes on the LightCycler 480 system (Roche Diagnostics). Following $2 \mathrm{~min}$ at $95^{\circ} \mathrm{C}, 40$ cycles were run, each with $3 \mathrm{sec}$ at $95^{\circ} \mathrm{C}$, $5 \mathrm{sec}$ at $60^{\circ} \mathrm{C}, 25 \mathrm{sec}$ at $60^{\circ} \mathrm{C}$, followed by melting curve analysis $\left(40^{\circ} \mathrm{C}\right.$ to $95^{\circ} \mathrm{C}$ ) after the PCR cycles (Promega GoTaq Probe PCR Master kit). The MACC1-specific PCR product of $136 \mathrm{bp}$ was amplified with the following primers (BioTeZ) and probes (TIB MolBiol): Forward primer, 5'-TTCTTTTGATTCCTCCGGTGA-3' and reverse primer, 5'-ACTCTGATGGGCATGTGCTG-3'; FITC-probe, 5'-GCAGACTTCCTCAAGAAATTCTGGAAGATCTA-3'; LCRed640-probe, 5'-AGTGTTTCAGAACTTCTGGAC ATTTTAGACGA-3'. MACC1 mRNA levels are presented as a percentage of the calibrator sample mRNA levels. The calibrator cDNA originated from the CRC cell line, SW620 (authentication by short tandem repeat genotyping, DSMZ). Serial dilutions of this calibrator RNA were employed for standard curve generation simultaneously in each quantitative PCR run. The in-run standard curve ranged from $100 \%$ calibrator down to $6.25 \%$ calibrator sample. Each sample was run and calculated in duplicate, the means are depicted.

MetScan. For the determination of circulating cell-free MACC1 in the plasma of individuals from MetScan, digital droplet PCR was performed using a Bio-Rad QX200 Droplet Digital PCR System (Bio-Rad Laboratories GmbH) and droplet digital polymerase chain reaction (ddPCR) Supermix for Probes (Bio-Rad Laboratories, Inc.) following the manufacturer's instructions (34). Briefly, $1 \mathrm{X}$ master mix was supplemented with primer probe mix (qHsaCPE5056798, Bio-Rad Laboratories, Inc.) and cDNA. Droplet generation for ddPCR was performed using the QX200 droplet generator. A total of $20 \mu \mathrm{l}$ PCR mix and $70 \mu 1$ droplet generator oil are given in the respective cavities of the droplet generator cartridges (Bio-Rad Laboratories, Inc.). Subsequently, $40 \mu 1$ droplet suspension was pipetted into twin.tec 96-well PCR plates (Eppendorf). PCR was performed at $95^{\circ} \mathrm{C}$ for $10 \mathrm{~min}$, by $39 \mathrm{x} 95^{\circ} \mathrm{C}$ for $30 \mathrm{sec}$ and $60^{\circ} \mathrm{C}$ for $60 \mathrm{sec}$, followed by $98^{\circ} \mathrm{C}$ for 10 min using a T100 thermal cycler (Bio-Rad Laboratories, Inc.). Droplet quantification was performed in the QX200 droplet reader. The system counts all generated droplets and detects the amount of PCR product-positive (fluorescent) droplets. The determination of circulating cell-free MACC1 transcripts was not successful in 13 out of the 204 MetScan participants, thus, 191 MetScan participants were included in the following analyses. The Poisson correction of generated 
droplet amount and data analysis was performed using the QUANTA LIFE (Bio-Rad Laboratories, Inc.) software.

Statistical analyses. For the animal experiments, statistical analyses were performed using Graph Pad Prism software V7 (GraphPad Software, Inc.). Statistical analyses were performed using a Student's t-test to compare results between the HFD-group with the appropriate control group. Spearman's correlation analysis was used to investigate the correlation between rat body weight and relative $\mathrm{MACC} 1$ expression. Values are presented as the mean \pm standard error of the mean (SEM). A value of $P \leq 0.05$ was considered to indicate a statistically significant difference.

For the MLU study, statistical analyses were performed using Graph Pad Prism software V7 (GraphPad Software, Inc.). Spearman's correlation analysis was used to investigate the correlation between MACC1 expression and body fat mass prior to the start of the intervention. The change in $\mathrm{MACC1}$ expression and body fat mass before and after intervention was determined by the subtraction of the values at 3 months after the start of the intervention from the values before the start of the intervention. Spearman's correlation analysis was used to determine the correlation between the changes in MACC1 expression and the changes in body fat mass by intervention. A value of $\mathrm{P} \leq 0.05$ was considered to indicate a statistically significant difference.

For the MetScan study, descriptive data on age, anthropometric and blood pressure measures are presented as the median and interquartile range (IQR), the frequency of MetS components and elevated MACC1 levels, WHR and WHtR as relative figures. Laboratory parameters are presented as the geometric mean (GM) and $95 \%$ confidence interval (CI). The level of circulating cell-free MACC1 transcripts was determined as 0.0 copies $/ 20 \mu 1$ in 27 out of the 191 participants. To avoid missing values for the log-transformed MACC1 measures for the respective participants, half of the minimal value $>0.0$ measured for MACC1 expression in the MetScan study population (i.e., 1.2 copies $/ 20 \mu \mathrm{l}$ ) was determined and this value was used to set MACC1 values of 0.0 as 0.6 copies $/ 20 \mu 1$. The respective participants were included with the modified values in the following analyses requiring a log-transformed value of MACC1 expression. Basic characteristics of participants were additionally analyzed by MACC1 tertiles.

Tests for differences across and between groups were analyzed using ANOVA and the post hoc Scheffe test or Kruskal-Wallis tests and the post hoc rank-based Scheffe test for continuous variables and Chi-squared tests for discrete variables. The mean level of circulating cell-free MACC1 transcripts (copies/20 $\mu \mathrm{l}$ ) was calculated according to the three BMI categories, first as raw estimates (presented as the median and IQR) and secondly based on least square means and log-transformed MACC1 values, adjusted for sex and age (presented as the GM and 95\% CI).

Additionally, the association of MACC1 expression with anthropometric measures, i.e., WC, BMI, WHR and WHtR, as well as metabolic parameters, i.e., glucose and HbAlc concentration and absolute fat mass, was investigated using Spearman's partial correlations adjusted for sex and age.

$\mathrm{P}$-values presented are two-tailed, with $\mathrm{P}<0.05$ considered to indicate a statistically significant difference. Analyses were performed using SAS ${ }^{\circledR}$ Enterprise Guide ${ }^{\circledR}$ (version 7.15; SAS Institute Inc.)

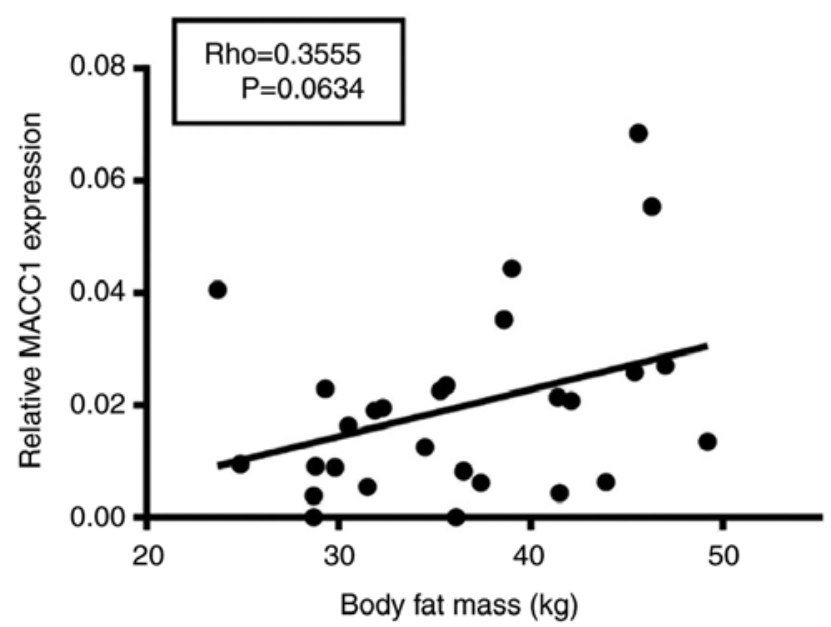

Figure 1. Relative expression of circulating cell-free plasma MACC1 transcripts and body fat mass in healthy obese adults $(n=29)$. Correlation analysis of relative MACC1 expression in plasma of healthy obese adults of the control as well as of the experimental group with the individual body fat mass. MACC1, metastasis-associated in colon cancer 1 .

\section{Results}

Correlation between circulating cell-free plasma MACC1 expression and body fat mass in the MLU study. First, the correlation between anthropometric parameters and MACC1 expression in plasma samples of obese human subjects was analyzed. Of note, a modest positive correlation between body fat mass and MACC1 expression $(\mathrm{Rho}=0.36)$ was found, which, however, was not significant at the $5 \%$ level $(\mathrm{P}=0.06$, Fig. 1).

Association of circulating cell-free plasma MACCl expression with anthropometric parameters in the MetScan study. Subsequently, MACC1 expression was measured in MetScan, a larger, independent study (Table I). The median MACC1 expression in MetScan was 3.4 copies/20 $\mu \mathrm{l}$ (IQR, 1.6-6.8) and GM 3.7 copies/20 $\mu \mathrm{l}$ (95\% CI, 3.1-4.5). When stratified by sex, the median expression in males was 4.0 copies/20 $\mu \mathrm{l}$ (IQR, 1.6-6.8) and GM was 4.0 copies/20 $\mu 1$ (95\% CI, 3.1-5.3), and in females the median expression was 3.1 copies/20 $\mu \mathrm{l}$ (IQR, 1.4-6.6) and GM 3.4 copies/ $20 \mu 1$ (95\% CI, 2.7-4.4); the differences between sexes were not statistically significant $(\mathrm{P}=0.28$ and $\mathrm{P}=0.40$, respectively).

The characteristics of the MetScan participants per tertiles of MACC1 expression are presented in Table II. Age and sex did not vary substantially across the MACC1 tertiles. However, there were differences in WC $(\mathrm{P}=0.02)$ and WHtR $(\mathrm{P}=0.03)$ across MACC1 tertiles with higher anthropometric measures in the upper than in the lower tertile. Similar, yet non-significant differences were observed for $\mathrm{BMI}(\mathrm{P}=0.06)$ and fat mass $(\mathrm{P}=0.07)$.

In unadjusted analyses, median MACC1 expression was 3.2 copies/20 $\mu \mathrm{l}$ (IQR, 1.6-6.4) among normal weight individuals (BMI $<25 \mathrm{~kg} / \mathrm{m}^{2}$ ), 3.0 copies/20 $\mu \mathrm{l}$ (IQR 1.4-4.6) among pre-obese individuals (BMI $25.0-29.9 \mathrm{~kg} / \mathrm{m}^{2} ; \mathrm{P}=0.93$ in comparison to normal weight) and 4.8 copies $/ 20 \mu \mathrm{l}$ (IQR 1.7-11.0) among obese individuals (BMI $\geq 30.0 \mathrm{~kg} / \mathrm{m}^{2} ; \mathrm{P}=0.16$ in comparison to normal weight; $\mathrm{P}=0.05$ for differences in MACC1 across BMI categories) (Table III). The results were similar when after adjusting for age and sex: 
Table I. Characteristics of the participants of the MetScan study $(\mathrm{n}=191)$.

\begin{tabular}{|c|c|c|c|}
\hline Characteristic & Total $(n=191)$ & Male $(n=91)$ & Female $(n=100)$ \\
\hline Age, years, median (IQR) & $54.2(40.3-66.3)$ & $55.2(42.0-67.5)$ & $54.1(38.0-65.3)$ \\
\hline Height, cm, median (IQR) & $172.0(166.1-179.7)$ & $179.3(174.3-183.3)$ & $166.8(161.6-170.1)$ \\
\hline WC, cm, median (IQR) & $95.2(83.6-105.1)$ & $98.0(89.5-109.6)$ & $92.6(79.8-102.6)$ \\
\hline BMI, kg/m², median (IQR) & $26.8(23.9-31.1)$ & $26.1(23.8-30.7)$ & $27.4(24.0-31.9)$ \\
\hline \multicolumn{4}{|l|}{ BMI categories, $\%$} \\
\hline$<25.0 \mathrm{~kg} / \mathrm{m}^{2}$ & 33.5 & 36.0 & 31.0 \\
\hline $25.0-29.9 \mathrm{~kg} / \mathrm{m}^{2}$ & 33.0 & 34.0 & 32.0 \\
\hline$\geq 30.0 \mathrm{~kg} / \mathrm{m}^{2}$ & 33.5 & 30.0 & 37.0 \\
\hline WHR, median (IQR) & $0.90(0.83-0.96)$ & $0.95(0.90-1.02)$ & $0.85(0.78-0.91)$ \\
\hline WHtR, median (IQR) & $0.55(0.49-0.62)$ & $0.54(0.49-0.62)$ & $0.56(0.48-0.62)$ \\
\hline Fat mass, kg, median (IQR) & $27.0(19.6-35.2)$ & $23.0(17.4-32.1)$ & $30.0(24.2-39.2)$ \\
\hline SBP, mmHg, median (IQR) & $122.0(114.5-135.5)$ & $128.5(119.5-141.0)$ & $117.0(109.5-127.0)$ \\
\hline DBP, mmHg, median (IQR) & $76.5(71.0-82.0)$ & $78.0(74.0-84.0)$ & $74.0(68.0-79.3)$ \\
\hline TG, mmol/1, GM (95\% CI) & $1.2(1.1-1.3)$ & $1.3(1.2-1.4)$ & $1.1(1.0-1.2)$ \\
\hline HDL-C, mg/dl, GM (95\% CI) & $1.5(1.4-1.5)$ & $1.3(1.2-1.4)$ & $1.6(1.6-1.7)$ \\
\hline Glucose, mmol/l, GM (95\% CI) & $5.9(5.8-6.0)$ & $6.1(5.9-6.2)$ & $5.7(5.6-5.9)$ \\
\hline HbA1c, mmol/mol, GM (95\% CI) & $35.7(35.0-36.5)$ & $35.6(34.5-36.8)$ & $35.9(34.8-37.0)$ \\
\hline Diabetes mellitus ${ }^{\mathrm{b}}, \%$ & 8.9 & 9.9 & 8.0 \\
\hline Dyslipidemia, \% & 36.1 & 28.6 & 43.0 \\
\hline MetS (meeting $\geq 3$ of the following five symptoms), $\%$ & 41.9 & 42.9 & 41.0 \\
\hline Elevated $\mathrm{WC}^{\mathrm{c}}, \%$ & 66.0 & 56.0 & 75.0 \\
\hline Elevated $\mathrm{TG}^{\mathrm{d}}, \%$ & 22.0 & 27.5 & 17.0 \\
\hline Reduced HDL-C,$\%$ & 8.9 & 5.5 & 12.0 \\
\hline Elevated blood pressure ${ }^{\mathrm{f}}, \%$ & 50.3 & 57.1 & 44.0 \\
\hline Elevated glucose ${ }^{\mathrm{g}}, \%$ & 66.0 & 76.9 & 56.0 \\
\hline MACC 1, copies/20 $\mu 1$, median (IQR) & $3.4(1.6-6.8)$ & $4.0(1.6-6.8)$ & $3.1(1.4-6.6)$ \\
\hline MACC 1, copies $/ 20 \mu 1^{\mathrm{a}}, \mathrm{GM}(95 \% \mathrm{CI})$ & $3.7(3.1-4.5)$ & $4.0(3.1-5.3)$ & $3.4(2.7-4.4)$ \\
\hline Elevated MACC $1, \%$ & 8.9 & 8.8 & 9.0 \\
\hline
\end{tabular}

Information on age and history of diabetes, dyslipidemia, and cancer was derived from self-reports during a personal interview; manual anthropometric, blood pressure as well as fasting blood samples were taken by trained personnel. a MACC1 value was 0 for 27 of the total 191 participants. To calculate the GM (95\%) MACC1 values were set as 0.6 copies $/ 20 \mu 1$ (half of the minimal value $>0$ measured for MACC1) for these participants and included the respective participants with the modified values in this analysis. ${ }^{b}$ Self-report of ever physician-diagnosed disease; 'males, $>94 \mathrm{~cm}$; women, $>80 \mathrm{~cm} \mathrm{(33);}{ }^{\mathrm{d}} \geq 1.7 \mathrm{mmol} / 1$ (33); ${ }^{\mathrm{e}}$ males, $<1.0 \mathrm{mmol} / \mathrm{l}$; women, $<1.3 \mathrm{mmol} / \mathrm{l}$ (33); ${ }^{\mathrm{f}}$ systolic blood pressure $\geq 130 \mathrm{mmHg}$ or mean diastolic blood pressure $\geq 85 \mathrm{~mm} \mathrm{Hg}$ (mean of the last two out of three sitting blood pressure measurements) or reported diagnosis of hypertension (33); ${ }^{\mathrm{g}} \geq 5.6 \mathrm{mmol} / \mathrm{l}$ or reported medical history of diabetes (33). BMI, body mass index; DBP, diastolic blood pressure; GM, geometric mean; HDL-C, high-density lipoprotein cholesterol; IQR, interquartile range; MACC1, metastasis-associated in colon cancer 1; MetS, Metabolic Syndrome; SBP, systolic blood pressure; TG, triglycerides; WC, waist circumference; WHR, waist-to-hip ratio; WHtR, waist-to-height ratio; 95\% CI, 95\% confidence interval.

Thus, geometric mean MACC1 levels were 3.3 copies $/ 20 \mu 1$ (95\% CI 2.4-4.5) among normal weight, 3.1 copies $/ 20 \mu 1$ (95\% CI 2.3-4.3) among pre-obese $(\mathrm{P}=0.98$ compared to normal weight), and 5.1 copies $/ 20 \mu 1$ (95\% CI 3.7-7.0) among obese individuals $(\mathrm{P}=0.18$ compared to normal weight; $\mathrm{P}=0.07$ across BMI categories; Fig. 2). The differences in MACC1 levels across BMI categories were slightly, although not-significantly $(\mathrm{P}=0.63)$ stronger among males than among females (Fig. 3).

When analyzed continuously, there were weak associations found between MACC1 and markers of general or abdominal adiposity and with markers of glucose metabolism (Table IV). For example, the Spearman's partial correlation coefficient for the association with MACC1 expression, adjusted for age and sex, was $0.10(\mathrm{P}=0.17)$ for $\mathrm{BMI}, 0.13(\mathrm{P}=0.07)$ for fat mass, 0.11 $(\mathrm{P}=0.12)$ for $\mathrm{WC}$ and $0.07(\mathrm{P}=0.36)$ for glucose levels. Of note, when stratified by sex, these associations were slightly stronger among males than among females (Table IV), although most correlations were not significant at the $5 \%$ level.

Association of circulating cell-free plasma MACC1 expression and body fat mass in obese individuals following dietary and exercise intervention in the MLU study. Previous results of the MLU study demonstrated that a moderate dietary and physical exercise program in obese individuals led to a significantly reduced body fat mass and waist and hip circumferences in the male sub-cohort following 3 months of intervention. 
Table II. Basic characteristics of the participants of the MetScan study by MACC1 tertiles.

MACC1 tertile

\begin{tabular}{|c|c|c|c|c|}
\hline \multirow{2}{*}{ Characteristic } & & & & \\
\hline & $1(\mathrm{n}=63)$ & $2(n=63)$ & $3(n=65)$ & P-value \\
\hline MACC1 copies/20 $\mu 1$ & $\leq 2.4$ & $>2.4$ to $\leq 4.8$ & $>4.8$ & \\
\hline Women, $\%$ & 58.7 & 49.2 & 49.2 & 0.47 \\
\hline Age, years, median (IQR) & $54.2(40.6-66.0)$ & $54.0(36.4-65.1)$ & $56.4(45.5-66.6)$ & 0.52 \\
\hline Height, cm, median (IQR) & $170.2(166.1-177.9)$ & $172.2(167.0-179.7)$ & $174.4(165.4-181.1)$ & 0.73 \\
\hline Waist circumference, $\mathrm{cm}$, median (IQR) & $92.7(82.8-103.9)$ & $92.7(84.5-102.3)$ & $99.5(88.5-111.6)$ & 0.02 \\
\hline BMI, kg/m², median (IQR) & $25.8(23.6-31.0)$ & $26.8(23.7-29.5)$ & $28.2(24.2-33.1)$ & 0.06 \\
\hline WHR, median (IQR) & $0.90(0.82-0.96)$ & $0.90(0.82-0.95)$ & $0.91(0.84-1.00)$ & 0.14 \\
\hline WHtR, median (IQR) & $0.54(0.48-0.60)$ & $0.53(0.49-0.60)$ & $0.58(0.50-0.64)$ & 0.03 \\
\hline Fat mass, kg, median (IQR) & $25.4(17.2-32.3)$ & $27.1(19.6-32.4)$ & $32.1(20.7-37.6)$ & 0.07 \\
\hline Systolic blood pressure, $\mathrm{mm} \mathrm{Hg}$ & $124.0(116-130.5)$ & $122.0(113.0-141.0)$ & $122.0(113.5-136.5)$ & 0.62 \\
\hline Diastolic blood pressure, $\mathrm{mm} \mathrm{Hg}$ & $76.0(71.5-81.5)$ & $77.5(72-87.5)$ & $76.5(70.0-81.0)$ & 0.07 \\
\hline $\mathrm{TG}, \mathrm{mmol} / \mathrm{l}, \mathrm{GM}(95 \% \mathrm{CI})$ & $1.15(1.02-1.28)$ & $1.15(1.03-1.29)$ & $1.25(1.12-1.40)$ & 0.31 \\
\hline HDL-C, mg/dl, GM (95\% CI) & $1.47(1.39-1.56)$ & $1.48(1.40-1.57)$ & $1.43(1.35-1.52)$ & 0.71 \\
\hline glucose, mmol/l, GM (95\% CI) & $5.75(5.57-5.95)$ & $5.85(5.66-6.05)$ & $6.04(5.84-6.24)$ & 0.27 \\
\hline $\mathrm{HbAlc}, \mathrm{mmol} / \mathrm{mol}, \mathrm{GM}(95 \% \mathrm{CI})$ & $35.7(34.3-37.1)$ & $34.8(33.5-36.2)$ & $36.8(35.4-38.2)$ & 0.10 \\
\hline Diabetes mellitus $^{\mathrm{a}}, \%$ & 7.9 & 4.8 & 13.8 & 0.19 \\
\hline Dyslipidemia $^{a}, \%$ & 31.7 & 36.5 & 40.0 & 0.62 \\
\hline MetS (meeting $\geq 3$ of the following five symptoms), $\%$ & 41.3 & 31.7 & 52.3 & 0.06 \\
\hline Elevated $\mathrm{WC}^{\mathrm{b}}, \%$ & 63.5 & 65.1 & 69.2 & 0.78 \\
\hline Elevated $\mathrm{TG}^{\mathrm{c}}, \%$ & 25.4 & 15.9 & 24.6 & 0.36 \\
\hline Reduced HDL-C ${ }^{\mathrm{d}}, \%$ & 11.1 & 4.8 & 10.8 & 0.37 \\
\hline Hypertension ${ }^{\mathrm{e}}, \%$ & 44.4 & 49.2 & 56.9 & 0.36 \\
\hline Elevated glucose $^{\mathrm{f}}, \%$ & 63.5 & 65.1 & 69.2 & 0.78 \\
\hline
\end{tabular}

Information on age and history of diabetes, dyslipidemia, and cancer was derived from self-reports during a personal interview; manual anthropometric, fat mass, blood pressure as well as fasting blood samples were taken by trained personnel. a Self-reported physician-diagnosed disease; ${ }^{b}$ males, $>94 \mathrm{~cm}$; women, $>80 \mathrm{~cm} \mathrm{(33);}{ }^{\mathrm{c}} \geq 1.7 \mathrm{mmol} / \mathrm{l}$ (33); ${ }^{\mathrm{d}}$ males, $<1.0 \mathrm{mmol} / \mathrm{l}$; women, $<1.3 \mathrm{mmol} / 1$ (33); ${ }^{\mathrm{e}}$ systolic blood pressure $\geq 130 \mathrm{mmHg}$ or mean diastolic blood pressure $\geq 85 \mathrm{~mm} \mathrm{Hg}$ (mean of the last two out of three sitting blood pressure measurements) or reported diagnosis of hypertension (33); ${ }^{\mathrm{f}} \geq 5.6 \mathrm{mmol} / \mathrm{l}$ or reported medical history of diabetes (33). BMI, body mass index; DBP, diastolic blood pressure; GM, geometric mean; HbA1c, HDL-C, high-density lipoprotein cholesterol; IQR, interquartile range; MACC1, metastasis-associated in colon cancer 1; MetS, Metabolic Syndrome; SBP, systolic blood pressure; TG, triglycerides; WC, waist circumference; WHR, waist-to-hip ratio; WHtR, waist-to-height ratio; 95\% CI, 95\% confidence interval.

Moreover, blood glucose levels significantly decreased in the male sub-cohort following intervention. The body weight and plasma concentrations of cholesterol, its subsets and triacylglycerols were reduced, but results were not significant (26). The results of the present analyses demonstrated that the change in body fat mass reduction negatively correlated with the change of MACC1 expression following three month of dietary intervention and physical exercise program, although results were not statistically significant $(\mathrm{P}=0.0698$, Fig. 4).

Circulating cell-free plasma MACCl expression in Wistar rats fed a NFD or HFD with or without CRC. Previous research has demonstrated that Wistar rats fed a HFD for 46 weeks exhibited significantly higher body weights and visceral fat mass compared to Wistar rats fed a NFD (28). In the present study, representative images of NFD- and HFD-fed Wistar rats are presented in Fig. 5A. Moreover, former investigations in diet-induced obese rats revealed a more severe tumor outcome with an increased number, size, and weight of colorectal tumors and a higher rate of adenocarcinomas than adenomas compared to the normal-weight control group (28). The results of the present analyses revealed no significant changes in circulating cell-free plasma MACC1 expression levels of rats fed the NFD compared with rats fed the HFD (Fig. 5B).

However, in AOM-exposed Wistar rats, plasma MACC1 expression was significantly increased in the HFD group compared with the NFD group $(\mathrm{P}<0.0001$, Fig. $6 \mathrm{~A})$. In addition, correlation analyses revealed a slight, although not significant, positive correlation between body weight and MACC1 expression in AOM-exposed rats $(\mathrm{P}=0.06$, Fig. $6 \mathrm{~B})$.

\section{Discussion}

Obesity is a major risk factor for the development of CRC, as well as for metastasis and cancer-associated mortality $(10,12)$. Several pathophysiological mechanisms for an increased risk 
Table III. Unadjusted median MACC1 expression in normal weight, pre-obese, and obese persons.

\begin{tabular}{|c|c|c|c|c|}
\hline \multirow[b]{2}{*}{ Group } & \multicolumn{2}{|c|}{$\begin{array}{l}\text { MACC } 1 \text { expression } \\
\quad(\text { copies } / 20 \mu 1)\end{array}$} & \multirow[b]{2}{*}{$\begin{array}{c}\text { P-value for differences } \\
\text { compared with reference group }\end{array}$} & \multirow[b]{2}{*}{$\begin{array}{c}\text { P-value for } \\
\text { difference across groups }\end{array}$} \\
\hline & Median & $\begin{array}{l}\text { Interquartile } \\
\text { range }\end{array}$ & & \\
\hline Normal weight $\left(\mathrm{BMI}<25.0 \mathrm{~kg} / \mathrm{m}^{2}\right)$ & 3.2 & $1.6-6.4$ & Reference & \\
\hline Pre-obese (BMI 25.0-29.9 kg/m²) & 3.0 & $1.4-4.6$ & 0.93 & 0.05 \\
\hline Obese $\left(\mathrm{BMI} \geq 30.0 \mathrm{~kg} / \mathrm{m}^{2}\right)$ & 4.8 & $1.7-11.0$ & 0.16 & \\
\hline
\end{tabular}

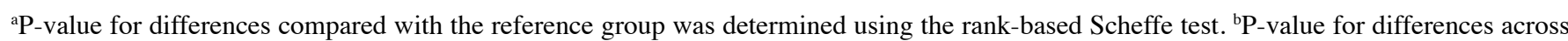
groups was determined using the Kruskal-Wallis test. BMI, body mass index; MACC1, metastasis-associated in colon cancer 1.

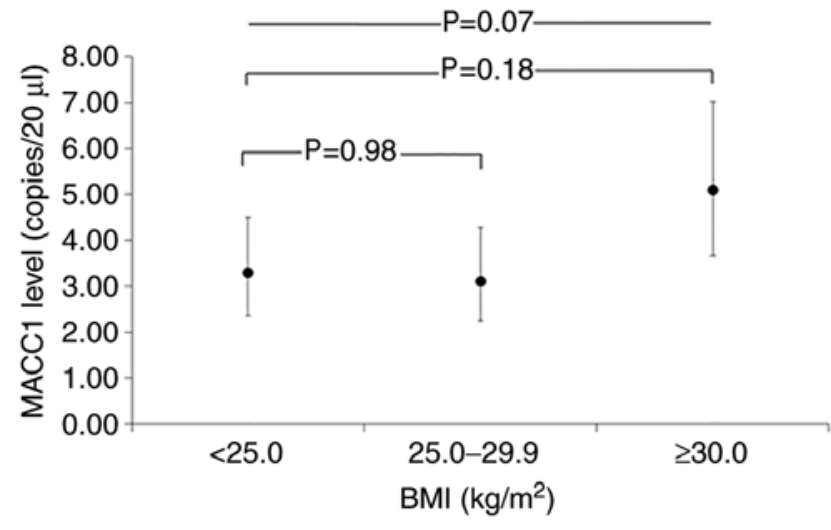

Figure 2. Geometric mean MACC1 levels (copies $/ 20 \mu 1$ ) and 95\% CI per BMI category, adjusted for sex and age in MetScan. MACC1 value was 0.0 copies $/ 20 \mu 1$ for 27 of the total 191 participants. To calculate the geometric mean $(95 \%)$ MACC1 values were set as 0.6 copies $/ 20 \mu$ l (half of the minimal value $>0.0$ measured for MACC1) for these participants and included the respective participants with the modified values in this analysis. Geometric mean MACC1 levels were 3.3 copies $/ 20 \mu 1$ (95\% CI, 2.4-4.5) among normal weight, 3.1 copies $/ 20 \mu 1$ (95\% CI, 2.3-4.3) among pre-obese ( $\mathrm{P}=0.98$ compared to normal weight), and 5.1 copies $/ 20 \mu \mathrm{l}(95 \% \mathrm{CI}, 3.7-7.0)$ among obese individuals $(\mathrm{P}=0.18$ compared to normal weight; $\mathrm{P}=0.07$ across $\mathrm{BMI}$ categories. Across-group comparisons are based on ANOVA and between-group comparisons are based on the Scheffe test, using log-transformed MACC1 concentrations. BMI, body mass index; MACC1, metastasis-associated in colon cancer 1 .

of CRC in obese individuals have been investigated. Apart from altered secretion profiles of adipocytokines, the increased release of growth factors and steroid hormones, oxidative stress and alterations in microbiome the chronic low-grade inflammation may contribute to the enhanced tumor development in obesity $(6,39-41)$. However, the underlying pathophysiological mechanisms linking obesity and cancer remain unclear.

MACC1 was discovered to be expressed in human CRC tissue $(13,14)$. Moreover, in vitro and in vivo studies have revealed the effects of MACC1 in tumor metabolism, proliferation, migration and invasion, and have demonstrated that MACC1 is a potent prognostic and predictive biomarker for several tumor entities, including CRC $(13,15,16,25)$. The present study, to the best of our knowledge, is the first to investigate the potential association between MACC1 expression, obesity and obesity-induced CRC.

The findings of the present study suggest an association between anthropometric parameters characterizing obesity
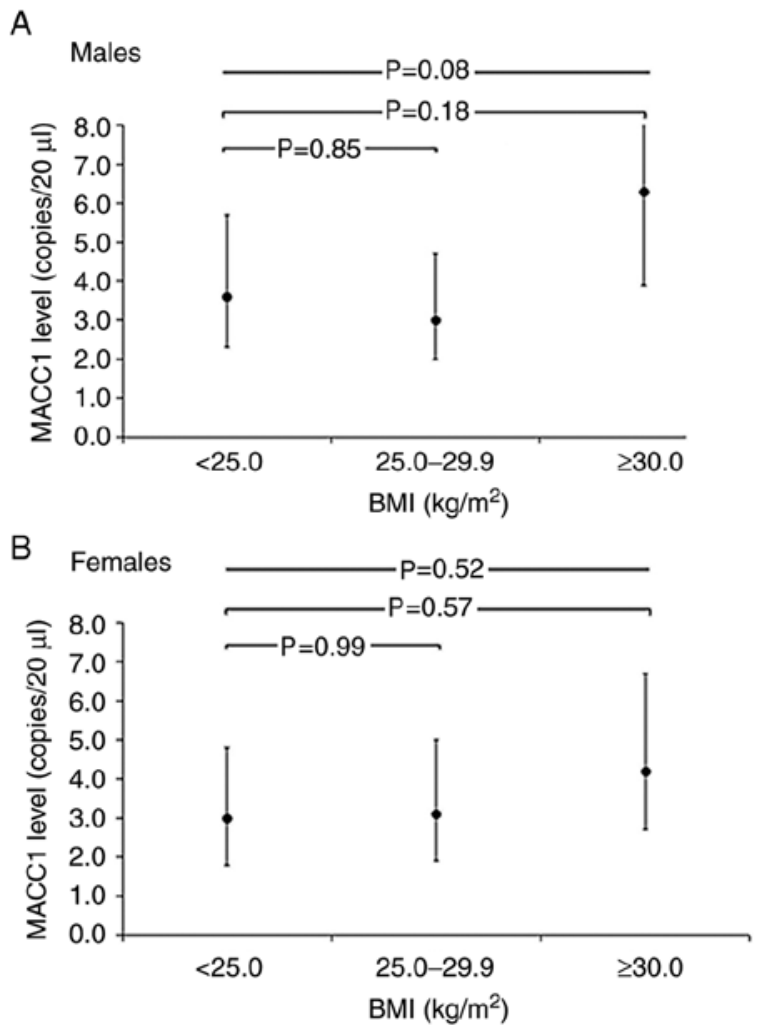

Figure 3. Geometric mean MACC1 levels (copies/20 $\mu \mathrm{l}$ ) and 95\% CI per BMI category, adjusted for age in MetScan. (A) Geometric mean MACC1 levels and $95 \%$ CI per BMI category, adjusted for age among males. Geometric mean MACC1 levels were 3.6 copies/20 $\mu 1$ (95\% CI, 2.3-5.7) among normal weight, 3.0 copies $/ 20 \mu 1$ (95\% CI, 2.0-4.7) among pre-obese ( $\mathrm{P}=0.85$ compared to normal weight) and 6.3 copies $/ 20 \mu \mathrm{l}$ (95\% CI, 3.9-10.1) among obese individuals $(\mathrm{P}=0.18$ compared to normal weight; $\mathrm{P}=0.08$ across $\mathrm{BMI}$ categories. (B) Geometric mean MACC1 levels and 95\% CI per BMI category, adjusted for age among females. Geometric mean MACC1 levels were 3.0 copies $/ 20 \mu 1$ (95\% CI, 1.8-4.8) among normal weight, 3.1 copies $/ 20 \mu 1$ (95\% CI, 1.9-5.0) among pre-obese $(\mathrm{P}=0.99$ compared to normal weight) and 4.2 copies $/ 20 \mu 1$ (95\% CI, 2.7-6.7) among obese individuals ( $\mathrm{P}=0.57$ compared to normal weight; $\mathrm{P}=0.52$ across $\mathrm{BMI}$ categories. The MACC1 value was 0.0 copies $/ 20 \mu 1$ for 27 of the total 191 participants. To calculate the geometric mean (95\%) MACC1 values were set as 0.6 copies $/ 20 \mu 1$ (half of the minimal value $>0.0$ measured for MACC1) for these participants and included the respective participants with the modified values in this analysis. Across-group comparisons are based on ANOVA and between-group comparisons are based on the Scheffe test, using log-transformed MACC1 concentrations. The test for difference in MACC1 levels across BMI categories was $\mathrm{P}=0.63$ ), based on $\mathrm{ANOVA}$ with the inclusion of an interaction term of sex x BMI categories. BMI, body mass index; MACC1, metastasis-associated in colon cancer 1 . 
Table IV. Spearman's partial correlation of anthropometric measures and biomarkers with MACC1 expression (copies/20 $\mu 1$ ).

\begin{tabular}{|c|c|c|c|c|c|c|}
\hline & \multicolumn{2}{|c|}{ Total $^{\mathrm{a}}(\mathrm{n}=191)$} & \multicolumn{2}{|c|}{$\operatorname{Male}^{\mathrm{b}}(\mathrm{n}=91)$} & \multicolumn{2}{|c|}{ Female $^{\mathrm{b}}(\mathrm{n}=100)$} \\
\hline & Rho & P-value & Rho & P-value & Rho & P-value \\
\hline BMI, $\mathrm{kg} / \mathrm{m}^{2}$ & 0.10 & 0.17 & 0.15 & 0.16 & 0.05 & 0.63 \\
\hline Fat mass, $\mathrm{kg}$ & 0.13 & 0.07 & 0.16 & 0.14 & 0.10 & 0.34 \\
\hline $\mathrm{WC}, \mathrm{cm}$ & 0.11 & 0.12 & 0.19 & 0.08 & 0.07 & 0.48 \\
\hline WHR & 0.05 & 0.48 & 0.20 & 0.06 & -0.03 & 0.79 \\
\hline WHtR & 0.14 & 0.05 & 0.19 & 0.07 & 0.12 & 0.25 \\
\hline Glucose, $\mathrm{mmol} / \mathrm{l}$ & 0.07 & 0.36 & 0.23 & 0.03 & -0.04 & 0.67 \\
\hline $\mathrm{HbA} 1 \mathrm{c}, \mathrm{mmol} / \mathrm{mol}$ & 0.07 & 0.36 & 0.20 & 0.07 & -0.04 & 0.71 \\
\hline
\end{tabular}

${ }^{a}$ Model adjusted for adjusted for age and sex; ${ }^{b}$ model adjusted for adjusted for age. BMI, body mass index; MACC1, metastasis-associated in colon cancer 1; WC, waist circumference; WHR, waist-to-hip ratio; WHtR, waist-to-height ratio.

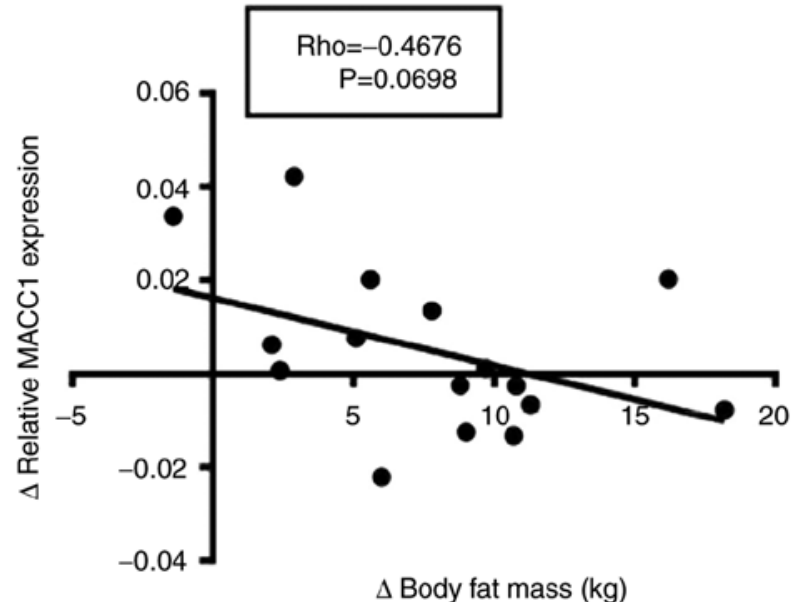

Figure 4. Relative expression of circulating cell-free plasma MACC 1 transcripts and body fat mass in obese individuals following dietary and exercise intervention. Correlation of the change in relative MACC1 expression in plasma of healthy obese adults of the experimental group with the individual change in body fat mass three months after start of intervention. MACC1, metastasis-associated in colon cancer 1.

and plasma MACC1 expression. Although not statistically significant, the results of the MLU cohort detected a slight positive correlation between body fat mass and plasma MACC1 expression in healthy obese subjects. In addition, analyses in MetScan demonstrated that participants in the highest MACC1 tertile had higher levels of BMI, WHtR, WC and fat mass, compared to participants in the lowest MACC1 tertile. Moreover, geometric mean MACC1 levels were the highest in the obese BMI group compared to normal weight and overweight participants. Although most of these associations were not statistically significant at the $5 \%$ level, these data of these two independent studies indicate that obesity may be associated with increased MACC1 expression levels. This is in line with the hypothesis that MACC1 expression may contribute to the higher risk for the development and metastasis of $\mathrm{CRC}$ in obese individuals.

Of note, when stratified by sex, the increased MACC1 levels in obesity were predominantly observed in male participants and to a lesser extent in female participants, although

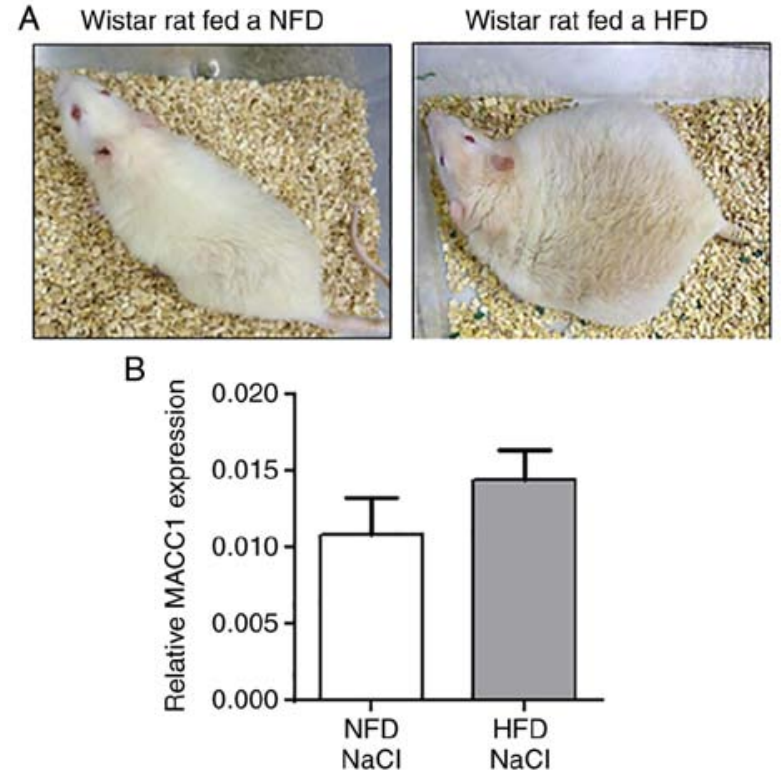

Figure 5. Relative expression of MACC1 in plasma of Wistar rats fed a NFD or a HFD and treated with a control solution $(\mathrm{NaCl})$. (A) Representative image of a Wistar rat fed the NFD or HFD for 46 weeks. (B) Relative MACC1 expression in the plasma of NFD- and HFD-fed Wistar rats. Data are expressed as the mean \pm SEM of $n=13$ (NFD) or 14 (HFD) animals per group. MACC1, metastasis-associated in colon cancer 1; NFD, normal-fat diet; $\mathrm{HFD}$, high-fat diet; $\mathrm{NaCl}$, sodium chloride.

these differences between males and females were not statistically significant. It remains to be elucidated whether possible sex-hormone-dependent effects in females of reproductive age may affect the MACC1 expression levels.

Previous studies have demonstrated an association between increased HbA1c or serum glucose levels and a higher cancer risk $(42,43)$. Although the insulin-insulin growth factor (IGF)-1 axis is a commonly suggested pathway, the mechanisms through which hyperglycemia contributes to an increased cancer risk are not yet completely understood.

The present study found a weak association between fasting glucose levels, as well as HbA1c and MACC1 levels in MetScan, and it may thus be hypothesized that MACC1 is involved in the pathway mediating the increased risk of cancer 
A

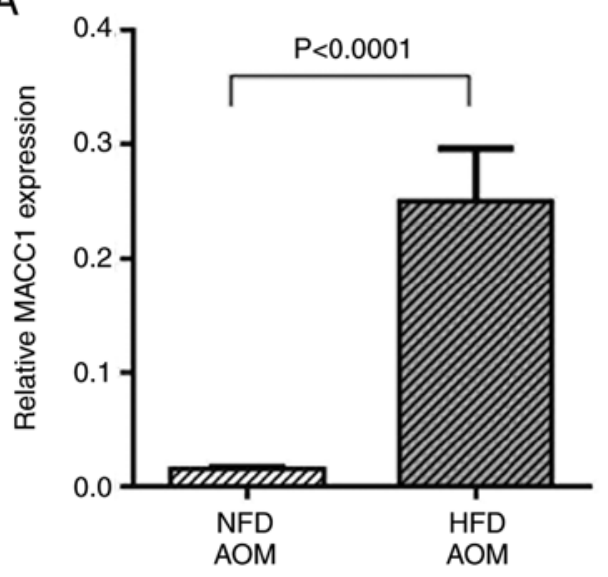

B

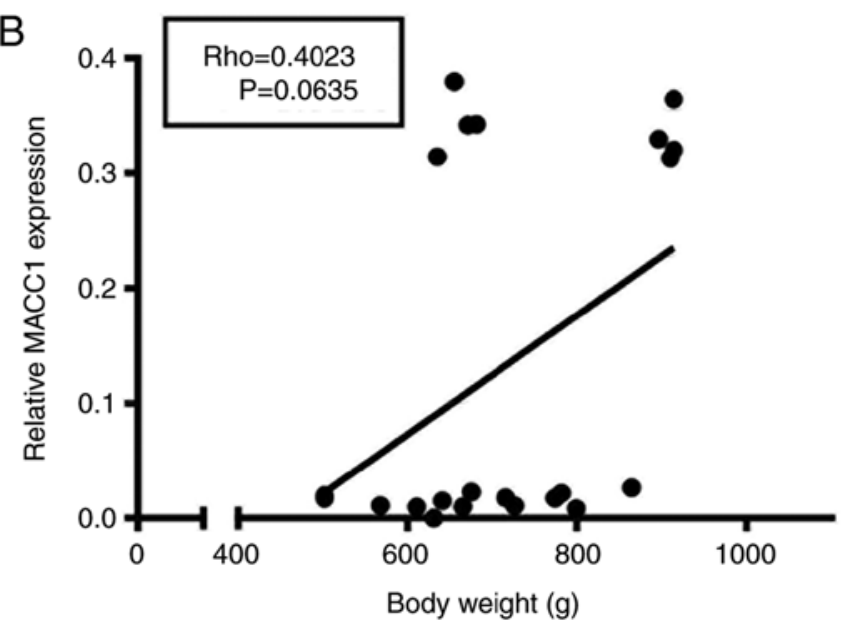

Figure 6. Relative expression of MACC1 in plasma and body weight of Wistar rats fed the NFD or HFD and treated with AOM. (A) Relative plasma MACC1 expression in AOM-treated Wistar rats fed the NFD or the HFD. Data are expressed as the mean \pm SEM of $n=11$ (HFD) or 12 (NFD) animals per group. $\mathrm{P}<0.001$, HFD group compared with the NFD group. (B) Correlation of the relative MACC1 expression in plasma of AOM-treated Wistar rats fed the NFD and HFD with the individual body weight of each rat $(n=23)$. MACC1, metastasis-associated in colon cancer 1; NFD, normal-fat diet; HFD, high-fat diet; AOM, azoxymethane.

and tumor metastasis by hyperglycemia; nevertheless, this association was statistically significant for glucose and only in males.

Although epidemiological studies investigating the loss of body weight and the subsequent cancer risk in obesity are limited, there is some evidence to indicate that a reduction in body weight can reduce the risk of cancer in obese individuals $(44,45)$. The present study analyzed whether a reduction in body weight and fat mass by a lifestyle intervention program in obese individuals may affect the plasma MACC1 expression levels. The results demonstrate that a change in body fat mass reduction negatively correlated with the change in $\mathrm{MACC} 1$ expression following 3 months of a moderate dietary intervention and physical exercise program. These data indicate that the increased MACC1 levels in obesity may not be normalized by a loss of body weight and fat mass or an enhanced level of physical activity. Apart from the significant decrease in fat mass in male participants, other anthropometric parameters such as BMI and body weight, were only moderately reduced after the 3-month intervention program of the MLU study (26). Therefore, further investigations are required to examine the impact of the loss of body weight or fat mass, as well as physical exercise on MACC1 levels in obese individuals.

The present study further analyzed the impact of obesity on plasma MACC1 expression in an animal model of CRC. Wistar rats treated with the carcinogen, AOM, exhibited a more severe colorectal tumor outcome when fed a HFD compared to AOM-treated rats fed a NFD (28). Notably, the results of the present study revealed that plasma MACC1 expression in AOM-exposed animals was significantly increased in rats fed the HFD compared to rats fed the NFD. These data indicate that the MACC1 levels may have contributed to the increased number, size and weight of colorectal tumors and a higher rate of adenocarcinomas than adenomas in HFD-fed rats exposed to AOM. Although no tumor metastases were detected in secondary organs of the AOM-exposed rats at the end of the experiment (28), it can be hypothesized that a higher metastasis rate in obese individuals with CRC may be associated by increased MACC1 levels and driven by MACC1-mediated pathways. Nevertheless, as AOM-exposed rats fed the HFD had a more severe colon tumor burden compared with AOM-exposed rats fed the NFD, future studies are warranted to investigate whether the high MACC1 levels in HFD-fed rats were induced by obesity or resulted from an increased MACC1 secretion by colon tumor cells. To date, the biological mechanisms through which obesity contributes to an increase of MACC1 remain unclear. Future studies are required to evaluate whether obesity-associated factors, such as alterations in adipocytokine levels or inflammatory parameters, may affect the expression of the transcription factor, MACC1. In addition, epidemiological long-term studies, including normal weight and obese patients with or without CRC are required to investigate whether increased MACC1 expression levels in obese individuals are associated with an increased risk of CRC and metastasis in humans. Moreover, further investigations are warranted to evaluate whether an association between the elevated MACC1 levels and the increased risk for tumor development in obesity may also be observed for other obesity-related cancer types, such as breast, liver, kidney or pancreatic cancer.

The present study has some limitations. Although MACC1 weakly correlated with anthropometric parameters of adiposity in the human studies, the majority of these results were statistically non-significant at the $5 \%$ level. As described above, in the MLU study, the correlation coefficient between body fat mass and MACC1 expression was $0.36(\mathrm{P}=0.06)$. Based on this observation, the MetScan sample was powered to detect correlation coefficients $r>0.2$ with adjustment for two covariates. The correlations that we observed in MetScan were $<0.2$ and statistically non-significant, which is in line with the current power calculations. When stratified by sex, slightly higher correlation coefficients were found among males than among females. These findings need to be interpreted cautiously, given that formal tests for interaction were statistically non-significant. In addition, one should also keep in mind that these latter were post hoc analyses, which included 
multiple testing, and that sample size was reduced in these stratified analyses.

In conclusion, the results of the present study revealed higher MACC1 plasma concentrations in obese as compared with non-obese persons, although these findings were not statistically significant. In addition, significantly increased plasma MACC1 expression levels were observed in obese rats exposed to AOM to induce colorectal tumors. These findings lead to the assumption that MACC1 is associated with pathophysiological pathways, contributing to an increased risk of $\mathrm{CRC}$ in obesity.

\section{Acknowledgements}

Not applicable.

\section{Funding}

MetScan was funded by the Federal Ministry for Economic Affairs and Energy (Bundesministerium für Wirtschaft und Energie, (BMWi), Förderkennzeichen KF2135010BZ4. The MACC1 association analyses were in part supported by the German Cancer Consortium (DKTK).

\section{Availability of data and materials}

The datasets used and/or analyzed during the current study are available from the corresponding author on reasonable request.

\section{Authors' contributions}

IB, HK, TP and US were involved in the conceptualization of the study. LJ, JJ, KN, DK and PH were involved in the study methodology. DK and PH were involved in the formal analysis, as well as in experimental plasma expression analyses. IB, LJ, TP and US were involved in the writing of the manuscript and in the preparation of the original draft. All authors were involved in the writing, reviewing and editing of the manuscript. IB, TP and US supervised the study. IB, TP, US and HK were involved in project administration. TP, US and HK were involved in funding acquisition. IB, HK, LJ, TP and US confirmed the authenticity of all the raw data. All authors have read and approved the final manuscript.

\section{Ethics approval and consent to participate}

All research and animal care procedures were approved by the local Animal Care Committee (reference number: 42502-2-1200 MLU). The principles of laboratory animal care were followed according to the guidelines of the European (FELASA) and German Society of Laboratory Animal Sciences (GV-SOLAS). For the MLU study, the study followed the Declaration of Helsinki on medical protocol and ethics and was approved by the ethics committee of the Faculty of Medicine, Martin Luther University Halle-Wittenberg (MLU), Halle (Saale), Germany. For the MetScan study, the study protocol was approved by the ethics committee of the Charité-Universitätsmedizin Berlin (reference no. EA1/197/15) and the local data protection officer. All investigations were carried out in accordance with the relevant guidelines and regulations, and written informed consent was obtained from all participants before inclusion. Informed consent was obtained from all subjects involved in the study.

\section{Patient consent for publication}

Not applicable.

\section{Competing interests}

The authors declare that they have no competing interests.

\section{References}

1. NCD Risk Factor Collaboration (NCD-RisC): Trends in adult body-mass index in 200 countries from 1975 to 2014: A pooled analysis of 1698 population-based measurement studies with 19.2 million participants. Lancet 387: 1377-1396, 2016.

2. World Health Organisation: Obesity and Overweight (WHO fact sheet). WHO, Geneva, Switzerland, 2021. https://www. who.int/news-room/fact-sheets/detail/obesity-and-overweight. Accessed December 22, 2021.

3. Robert Koch Institute: Overweight and Obesity. Robert Koch Institute, Berlin, Germany, 2021. https://www.rki.de/EN/ Content/Health_Monitoring/Main_Topics/Overweight_Obesity/ obesity_node.html;jsessionid=B0011790B0977853D808900F 073E7775.internet111. Accessed December 22, 2021.

4. Renehan AG, Tyson M, Egger M, Heller RF and Zwahlen M: Body-mass index and incidence of cancer: A systematic review and meta-analysis of prospective observational studies. Lancet 371: 569-578, 2008.

5. Ma Y, Yang Y, Wang F,Zhang P, Shi C, Zou Y and Qin H: Obesity and risk of colorectal cancer: A systematic review of prospective studies. PLoS One 8: e53916, 2013.

6. Dong Y, Zhou J, Zhu Y, Luo L, He T, Hu H, Liu H, Zhang Y, Luo D, Xu S, et al: Abdominal obesity and colorectal cancer risk: Systematic review and meta-analysis of prospective studies. Biosci Rep: Dec 12, 2017 (Epub ahead of print). doi: 10.1042/ BSR20170945.

7. Renehan AG and Soerjomataram I: Obesity as an avoidable cause of cancer (Attributable Risks). Recent Results Cancer Res 208: 243-256, 2016.

8. Sung H, Ferlay J, Siegel RL, Laversanne M, Soerjomataram I, Jemal A and Bray F: Global cancer statistics 2020: GLOBOCAN estimates of incidence and mortality worldwide for 36 cancers in 185 countries. CA Cancer J Clin 71: 209-249, 2021.

9. Larsson SC and Wolk A: Obesity and colon and rectal cancer risk: A meta-analysis of prospective studies. Am J Clin Nutr 86: 556-565, 2007.

10. Calle EF, Rodriguez C, Walker-Turmond $\mathrm{K}$ and Tun MJ: Overweight, obesity, and mortality from cancer in a prospectively studied cohort of U.S. Adults. New Engl J Med 348: 1625-1638, 2003.

11. Campbell PT, Newton CC, Dehal AN, Jacobs EJ, Patel AV and Gapstur SM: Impact of body mass index on survival after colorectal cancer diagnosis: The cancer prevention StudyII nutrition cohort. J Clin Oncol 30: 42-52, 2012.

12. Arnold M, Leitzmann M, Freisling H, Bray F, Romieu I, Renehan A and Soerjomataram I: Obesity and cancer: An update of the global impact. Cancer Epidemiol 41: 8-15, 2016.

13. Stein U, Walther W, Arlt F, Schwabe H, Smith J, Fichtner I, Birchmeier W and Schlag PM: MACC1, a newly identified key regulator of HGF-MET signaling, predicts colon cancer metastasis. Nat Med 15: 59-67, 2009.

14. Radhakrishnan H, Walther W, Zincke F, Kobelt D, Imbastari F, Erdem M, Kortüm B, Dahlmann M and Stein U: MACC1-the first decade of a key metastasis molecule from gene discovery to clinical translation. Cancer Metastasis Rev 37: 805-820, 2018.

15. Pichorner A, Sack U, Kobelt D, Kelch I, Arlt F, Smith J, Walther W, Schlag PM and Stein U: In vivo imaging of colorectal cancer growth and metastasis by targeting MACC1 with shRNA in xenografted mice. Clin Exp Metastasis 29: 573-583, 2012. 
16. Lemos C, Hardt MS, Juneja M, Voss C, Förster S, Jerchow B, Haider W, Bläker $\mathrm{H}$ and Stein U: MACC1 induces tumor progression in transgenic mice and colorectal cancer patients via increased pluripotency markers Nanog and Oct4. Clin Cancer Res 22: 2812-2824, 2016.

17. Wang G, Fu Z and Li D: MACC1 overexpression and survival in solid tumors: A meta-analysis. Tumour Biol 36: 1055-1065, 2015

18. Wu Z, Zhou R, Su Y, Sun L, Liao Y and Liao W: Prognostic value of macc1 in digestive system neoplasms: A systematic review and meta-analysis. Biomed Res Int 2015: 252043, 2015.

19. Sun DW, Zhang YY, Qi Y, Liu GQ, Chen YG, Ma J and Lv GY: Prognostic and clinicopathological significance of MACC1 expression in hepatocellular carcinoma patients: A meta-analysis. Int J Clin Exp Med 8: 4769-4777, 2015.

20. Zhao Y, Dai C, Wang M, Kang H, Lin S, Yang P, Liu X, Liu K, Xu P, Zheng Y, et al: Clinicopathological and prognostic significance of metastasis-associated in colon cancer-1 (MACC1) overexpression in colorectal cancer: A meta-analysis. Oncotarget 7: 62966-62975, 2016

21. Jin Y, Zhou K, Zhao W, Han R, Huo X, Yang $F$ and Chen J: Clinicopathological and prognostic significance of metastasis-associated in colon cancer- 1 in gastric cancer: A meta-analysis. Int J Biol Markers 34: 27-32, 2019.

22. Wang L, Fan L, Xu H and Jiang H: Prognostic significance of the expression of metastasis-associated in colon cancer-1 in gynecologic cancers and breast cancer: A protocol for systematic review and meta-analysis. Medicine (Baltimore) 100: e24255. 2021.

23. Li Y, Lu Z, Liang Z, Ji D, Zhang P, Liu Q, Zheng X and Yao Y: Metastasis-associated in colon cancer-1 is associated with poor prognosis in hepatocellular carcinoma, partly by promoting proliferation through enhanced glucose metabolism. Mol Med Rep 12: 426-434, 2015.

24. Zhang M, Yang J, Jiang H, Jiang $\mathrm{H}$ and Wang Z: Correlation between glucose metabolism parameters derived from FDG and tumor TNM stages and metastasis-associated proteins in colorectal carcinoma patients. BMC Cancer 21: 258, 2021.

25. Lisec J, Kobelt D, Walther W, Mokrizkij M, Grötzinger C, Jaeger C, Baum K, Simon M, Wolf J, Beindorff N, et al: Systematic identification of MACC1-driven metabolic networks in colorectal cancer. Cancers (Basel) 13: 978, 2021.

26. Jahn J, Spielau M, Brandsch C, Stangl GI, Delank KS, Bähr I, Berreis T, Wrann CD and Kielstein H: Decreased NK cell functions in obesity can be reactivated by fat mass reduction. Obesity (Silver Spring) 23: 2233-2241, 2015.

27. Jaeschke L, Steinbrecher A, Hansen G, Sommer S, Adler C Janke J and Pischon T: Association of body surface scanner-based abdominal volume with parameters of the Metabolic Syndrome and comparison with manually measured waist circumference. Sci Rep 10: 9324, 2020

28. Bähr I, Goritz V, Doberstein H, Hiller GGR, Rosenstock P, Jahn J, Pörtner O, Berreis T, Mueller T, Spielmann J and Kielstein H: Diet-induced obesity is associated with an impaired NK cell function and an increased colon cancer incidence. J Nutr Metab 2017: 4297025, 2017.

29. Bähr I, Pörtner OJ, Glass M, Doberstein H, Goritz V, Hiller GGR, Spielmann $\mathrm{J}$ and Kielstein $\mathrm{H}$ : Characterization of natural killer cells in colorectal tumor tissue of rats fed a control diet or a high-fat diet. Ann Anat 233: 151586, 2021.

30. Adler C, Steinbrecher A, Jaeschke L, Mähler A, Boschmann M, Jeran $\mathrm{S}$ and Pischon T: Validity and reliability of total body volume and relative body fat mass from a 3-dimensional photonic body surface scanner. PLoS One 12: e0180201, 2017.

31. World Health Organization: Waist Circumference and Waist-Hip Ratio.ReportofaWHOExpertConsultation.Geneva,8-11December 2008. World Health Organization, Geneva, 2011. Available from: http://www.who.int/nutrition/publications/obesity/WHO_report_waist circumference_and_waisthip_ratio/en/.
32. Schulze MB,Kroke A, Bergmann MM and Boeing H: Differences of blood pressure estimates between consecutive measurements on one occasion: Implications for inter-study comparability of epidemiologic studies. Eur J Epidemiol 16: 891-898, 2000.

33. Alberti KG, Eckel RH, Grundy SM, Zimmet PZ, Cleeman JI, Donato KA, Fruchart JC, James WP, Loria CM, Smith SC Jr, et al: Harmonizing the metabolic syndrome: A joint interim statement of the international diabetes federation Task Force on epidemiology and prevention; National heart, lung, and blood institute; American Heart Association; World Heart Federation; International atherosclerosis society; and International association for the study of obesity. Circulation 120: 1640-1645, 2009.

34. Hagemann C, Neuhaus N, Dahlmann M, Kessler AF, Kobelt D, Herrmann P, Eyrich M, Freitag B, Linsenmann T, Monoranu CM, et al: Circulating MACC1 transcripts in glioblastoma patients predict prognosis and treatment response. Cancers (Basel) 11: 825, 2019.

35. Link T, Kuhlmann JD, Kobelt D, Herrmann P, Vassileva YD, Kramer M, Frank K, Göckenjan M, Wimberger P and Stein U: Clinical relevance of circulating MACC1 and S100A4 transcripts for ovarian cancer. Mol Oncol 13: 1268-1279, 2019.

36. Ashktorab H, Hermann P, Nouraie M, Shokrani B, Lee E, Haidary T, Brim H and Stein U: Increased MACC1 levels in tissues and blood identify colon adenoma patients at high risk J Transl Med 14: 215, 2016.

37. Burock S, Herrmann P, Wendler I, Niederstrasser M, Wernecke KD and Stein U: Circulating Metastasis Associated in Colon Cancer 1 transcripts in gastric cancer patient plasma as diagnostic and prognostic biomarker. World J Gastroenterol 21: 333-341, 2015.

38. Stein U, Burock S, Herrmann P, Wendler I, Niederstrasser M, Wernecke KD and Schlag PM: Circulating MACC1 transcripts in colorectal cancer patient plasma predict metastasis and prognosis. PLoS One 7: e49249, 2012.

39. Ackerman SE, Blackburn OA, Marchildon F and Cohen P: Insights into the link between obesity and cancer. Curr Obes Rep 6: 195-203, 2017.

40. Berger NA: Obesity and cancer pathogenesis. Ann N Y Acad Sci 1311: 57-76, 2014.

41. Himbert C, Delphan M, Scherer D, Bowers LW, Hursting S and Ulrich CM: Signals from the adipose microenvironment and the obesity-cancer link-A systematic review. Cancer Prev Res (Phila) 10: 494-506, 2017.

42. Crawley DJ, Holmberg L, Melvin JC, Loda M, Chowdhury S, Rudman SM and Van Hemelrijck M: Serum glucose and risk of cancer: A meta-analysis. BMC Cancer 14: 985, 2014.

43. Hope C, Robertshaw A, Cheung KL, Idris I and English E: Relationship between HbAlc and cancer in people with or without diabetes: A systematic review. Diabet Med 33: 1013-1025, 2016.

44. Luo J, Hendryx M, Manson JE, Figueiredo JC, LeBlanc ES, Barrington W, Rohan TE, Howard BV, Reding K, Ho GY, et al: Intentional weight loss and obesity-related cancer risk. JNCI Cancer Spectr 3: pkz054, 2019.

45. Tee MC, Cao Y, Warnock GL, Hu FB and Chavarro JE: Effect of bariatric surgery on oncologic out-comes: A systematic review and meta-analysis. Surg Endosc 27: 4449-4456, 2013.

This work is licensed under a Creative Commons Attribution-NonCommercial-NoDerivatives 4.0 International (CC BY-NC-ND 4.0) License. 